\title{
A framework for participatory assessment of vulnerability of commercial property values to flooding in the UK
}

\author{
N. Bhattacharya ${ }^{1}$, J. Lamond ${ }^{2}$, D. G. Proverbs ${ }^{2}$, \\ F. Hammond ${ }^{1} \&$ D. Searle ${ }^{1}$ \\ ${ }^{1}$ School of Technology, University of Wolverhampton, UK \\ ${ }^{2}$ Faculty of Environment and Technology, \\ University of West of England, UK
}

\begin{abstract}
The characteristics of flood risk situation in the UK for commercial properties involve large scale complexity in terms of effective contributory variables, different levels of spatio-temporal scales, and affected stakeholders' involvement. In the process of risk assessment, vulnerability has emerged as one of the most critical concepts as an indicative factor of measurement of severity of the likely consequence. The study builds on evaluation of existing approaches of participatory vulnerability and valuation approaches and moves on to develop new pluralistic participatory vulnerability analysis (PVA) method for analysis of qualitative and quantitative information. The focus is to investigate the effectiveness of participatory vulnerability analysis (PVA) approach supported by GIS as a potential tool for the UK commercial property sector for assessment of vulnerability of property values towards flooding. This includes the involvement of affected stakeholders to evaluate the predictive effect of the contributory factors on vulnerability of commercial property values. The proposed participatory spatial analysis method is suitable to be implemented within a Geographic Information System (GIS) framework. The approach is simple and transparent allowing public participation as well as accommodating data gaps by including opinions and experiential knowledge in the analysis.

Keywords: commercial properties, GIS, PVA, spatial analysis, valuation.
\end{abstract}




\section{Introduction}

In England and Wales approximately 185,000 commercial properties are at risk of flooding and average damage attributed to inland flooding is approximately $£ 1$ Billion annually. Bennett [1] reported that this spending could exceed up to $£ 27$ Billion by 2080 .

As units of study in disaster research, commercial properties have recently made their place. Much of the research in the field is concentrated on residential properties and few have included empirical research of the commercial property sector [2]. Various traditional and modern methods of valuation are available which greatly depend on availability of market data. Each of these methods has their own advantages and disadvantages in relation to data availability and condition of usage. Pagourtzi et al. [3] and Shapiro et al. [4] noted that valuation studies of commercial properties in the UK have relied on knowledge and judgement of valuers. This is mainly because of difficulty in accessibility of valuation data at public domain and diverse and complex nature of data. Lack of data has affected valuation techniques and valuers often adopt options like available alternative comparable property data evidences [4, 5]. Spatial analysis method is one of the advanced methods which have a large potential in predictive modelling especially where data for comparative analysis of properties are sparsely available which the case in the UK.

Vulnerability is a dynamic concept which exposes the intrinsic feature of the community indicating a damage potential with multitude of components and forward looking variables (UNU-EHS personal communication, 2004 cited in Thywissen [6]). Vulnerability assessment for flood affected populations and properties are common in disaster research but are rarely used for commercial property valuation studies. It is highly relevant to have a comprehensive methodology to overview and analyse the potential impact flood vulnerability has on the value of commercial properties [7-9]. The motivation is to identify robust techniques that are sustainable to the changing physical and economic environment and reflect the market condition.

The term "vulnerability" of property is complex and involves a number of physical and social factors. Kenyon [10] reported that there are uncertainties involved in the assessment of vulnerability related to social perspective, where various stakeholders have conflicting interests, multiple legitimate perspectives, diffused responsibilities and differing level of impacts. Arguments have been put forward that under such complex circumstances, with variable criteria, a participatory approach is crucial for proper assessment and management $[11,12]$. There is also increased emphasis on the participatory role of local government and stakeholders in flood risk management. However the challenge is to find the appropriate method or combination of methods where participatory approaches can be utilized and can be most effective for specific objectives. The main focus of the research is to analyse the effectiveness of the application of participatory vulnerability analysis (PVA) process to UK commercial property sector as a potential tool for assessment of vulnerability of property values towards flooding. 
The paper is structured in three sections. First, description of the existing method of vulnerability assessment and Participatory Vulnerability Analysis is done. An evaluation of the methods is performed in the next section to recognize their suitability to the set objectives and identify potential enhancements. Based on this rationale the next section describes a novel method and the features of this new method that address issues identified associated with existing method. Finally, recommendation for implementation of the proposed method for vulnerability assessment of commercial properties in the UK is discussed before concluding.

\section{Description of existing methods}

\subsection{The concept of vulnerability assessment}

In the process of risk assessment, vulnerability has emerged as one of the most critical concepts as an indicative factor of measurement of severity of the likely consequence [13-15]. Vulnerability assessment has the advantage of involving multiple components to provide a holistic view of the level of risk and its value can be communicated among the stakeholders easily [16]. However, the number of indicators required for vulnerability assessment is highly data demanding and intensive in nature which often poses problem for the researchers. It is recognized that some of the criteria are either redundant or have no significant influence on the result.

Balica and Wright $[16,17]$ accounted that the number of indicators can be reduced to a manageable level based on the spatial scale of flood occurrence. Flood vulnerability assessment is useful if it is based on the most significant indicators with the focus on obtaining knowledge from the participation of stakeholders and allowing the end users to simplify their work as far as possible.

The actual factors are very site specific, location and hazard dependent [18]. The standard practice in this regard is to use the deductive approach, which is to follow a conceptual framework; this involves understanding the phenomenon followed by identification and selection of vulnerability indicators $[17,19,20]$.

This criterion of selection of preliminary suitable indicators has been adopted for the proposed methodology. The selection of criteria and their suitability to local conditions can be backed up by outcome of expert survey. As a result incorporation of participatory approaches in this process is crucial.

\subsection{Participatory approaches}

In terms of complex environmental problems such as flood risk management, where several stakeholders and large number of properties with high potential of damage are involved, participatory approaches are increasingly recommended [21-23].

Actionaid [18] defined the concept of Participatory vulnerability assessment (PVA) as a systematic approach with the involvement of stakeholders in a thorough analysis of their level of vulnerability to empower and motivate them 
for appropriate future actions of preparedness and response to long term development. Since the nature of vulnerability is complex, and it requires predictive judgement, the analysis needs detailed and to the point breakdown. This is possible by dividing the process into well-defined phases as shown in figure 1 .

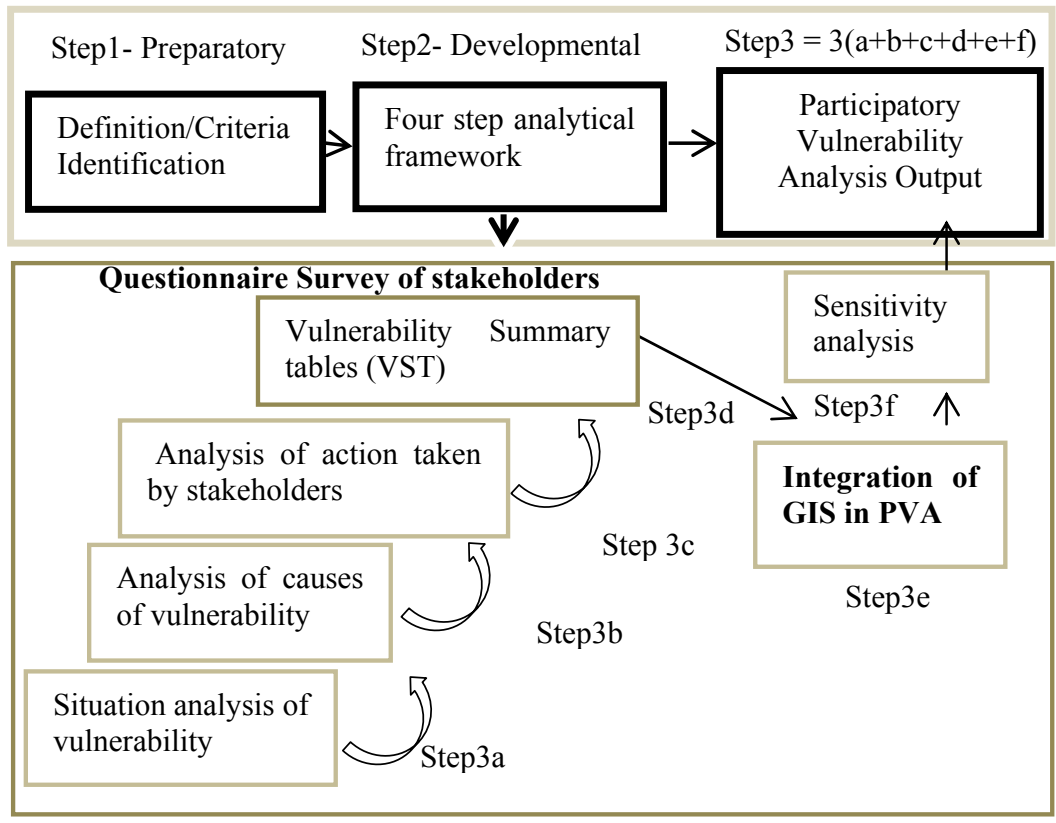

Figure 1: Participatory vulnerability analysis phases.

The stakeholders are given chance to weight the factors which are defined within the set questionnaire, according to their level of knowledge and experience. At the end of the participatory stage a complete matrix (vulnerability summary table or VST) of impact category, likelihood, impact details along with their weights should ideally be achieved to continue to the next stage. This phase will act as a base for the stages to follow where all these related information can be included in the generation of final composite map of vulnerability using Geographic Information System (GIS). Therefore, a spatial analysis technique supported by Participatory vulnerability analysis approach is proposed here as an appropriate methodology to assess the impact of flood vulnerability to commercial property value in the UK.

\section{Evaluation of the proposed approach}

The proposed new approach is designed to work with the local stakeholders involved in commercial property sector to conduct vulnerability assessment and identify the major drivers that affect property value in case of flood vulnerability. 
Research has confirmed that there is a need to embed participatory processes in vulnerability assessment to benefit fully from the process and its wider relevance in the UK commercial property sector. PVA approach has benefit of providing transparency to the participants in understanding the different contributory factors of vulnerability. Prabhu et al. [22] described the process... is considered to be "simple, intuitive and transparent" and has the ability to deal with both qualitative and quantitative data. It has further scope of including experiential knowledge within the database in case of non-availability of observational data; therefore PVA is selected as a particularly appropriate methodology especially for markets where data is scarce as is the case in the UK.

Concerns related to the reliance on the knowledge of stakeholders in weighting the factors affecting their level of vulnerability may arise. Appropriate weighting of different factors according to their level of impact is necessarily subjective because the relationships between different factors are not linear and there are number of uncertainties involved. However, Muller et al. [24] noted that incorporation of information from two different sources (literature and stakeholders) could be used to strengthen the evidence for judgements as to how the scores may be assigned. Sensitivity analysis of outputs could be the answer to understand differential impact of differently scored factors on the outputs. It becomes especially important to understand the transferability of results for different stakeholders' perception.

Spatial data was rarely used in previous researches in the field of vulnerability of property value especially in the UK. Some of the methods used previously had problems like narrow domain of selecting implicit number of comparable properties from immediate localities and lacked in exhibiting spatio-temporal knowledge representatively [25-27]. The spatial analysis method, on the other hand, has great analytical capacity with the use of spatial data and interpolation techniques. This approach provides potential for a 'value range' maps which can be utilized for comparable properties in the form of a surface determined by the best representative data using GIS.

GIS can be used where various complex factors are involved as a potentially important tool for analysing digital data in space and time. This is because GIS has the ability to combine information of various spatial nature and source in one platform and illustrate it in an easily understandable manner to the concerned stakeholders in the form of maps. Pagourtzi et al. [3] and Wyatt [5] emphasized the benefits of GIS in their research in generation of 'value maps' including spatial distribution of values along with other property attributes. They also stressed on the potential use of GIS interface in value factor selection, units of comparison and analysis.

Pagourtzi et al. [3] and Lamond [25] indicated that the UK has lack of statistically significant sample sizes, comparative sales data of properties, therefore technique like spatial analysis are useful in such case .The proposed approach has advantage over the other approaches as it allows to go beyond the traditional way of explaining impact on property value using a set of man-made and ecological variables but also incorporate spatial heterogeneity, frequent updating using updated digital data without the need for repeated survey and 
greater analytical potential in research using GIS. Wyatt [27] pointed out that when valuation attributes are combined with flood vulnerability maps incorporating participatory approaches they can prove to be powerful decision making tools . They are also useful for purposes like planning, taxation, asset management, zonation of high and low value zones of properties (with particular reference to flood risk), recording changes in valuation over time and in assisting future valuation $[5,27]$.

\section{Method design}

\subsection{Participatory Vulnerability Analysis (PVA)}

The method is partly designed based on the framework guided by Actionaid International [18]. The purpose of this research focuses on analysis of the level of vulnerability and their root causes within a certain vulnerable group i.e., the commercial property sector. The methodological approach contains a flow of criteria which is guided by problem identification with specified objectives, problem structuring through screening in the form of VST. The VSTs include information related to impact data, qualitative and quantitative in nature. Based on these VSTs, type and category of impacts are identified for different levels of vulnerability of properties. The impact types can be categorized into physical, economic and social; while the impact categories are not fixed they differ based on distinct nature of impact and characteristics of the area. A questionnaire formulation is necessary including all the factors and criterion within each category. Data for the VSTs are collected mainly based on the survey.

At this stage it is important to involve the concerned stakeholders for instance the property occupiers to gain information and knowledge which can be compared with the existing literature based on the work flow showed in figure 1. For this reason, it is useful to start with a pilot study or smaller group. This can help in ensuring that the design for assessment has taken into account the most important factors. This also provides an option for screening out those categories of factors which do not comply with the scope of the study.

The process of ranking is allocated within the questionnaire that enables the participants (stakeholders) to rank the criterion based on their own judgement and weights can be derived from this information at a later stage of analysis. The summary of the VST provides different options for analysis of the effect of level of flood vulnerability on properties. These options for analysis are important aspects for understanding the potential impact on valuation as they have a futuristic component which involves a predictive quality. The process of PVA provides the base for the next stage which is the integration of the gathered information to assign the level of vulnerability to a composite vulnerability map by integration of GIS.The novelty in the approach is that the method is used to assess vulnerability of property values based on their level of vulnerability identified through integration of participatory approaches. 


\subsection{Integration of GIS in the assessment of vulnerability analysis}

The contributing factors or selection of evaluation criteria for flood impact are categorized into physical, economic, social, technological and resilient attributes. These factors are taken into account in the frame of contribution towards vulnerability of flooding. Each factor is represented by a layer to form criteria maps which make the factors flexible to changes and highly transparent for the users to notice their effect on property values. Subsequently attributes are added to the layers to describe the information related to the causal factors.

Information obtained from the participatory approach are weighted according to their importance and added as layers for each contributing factor towards vulnerability. Muller et al. [24] described that to make the various maps comparable standardization of the ranked maps is necessary .Valuation data for commercial properties on a temporal basis are then formatted to be used in GIS environment. This data helps to create 'value range' map utilizing other attributes to illustrate the geographical distribution of property values over space and time. The value data is also helpful in spatial value pattern analysis, and autocorrelation analysis of property values. Such analysis can be a useful in predictive vulnerability assessment for property values where data is sparse.

\subsubsection{Sensitivity Analysis}

Sensitivity Analysis (SA) is a prerequisite for any model since that determines the reliability of the model in assessment of uncertainties. In this case, the different input parameters are systematically varied based on their weights provided by different groups of stakeholders to understand the change in behaviour of the outputs.

Edgar [26] emphasized that pairwise comparisons between most relevant factors can be useful for carrying out such analysis. It decomposes the problem in a hierarchical manner with comparative judgement and synthesis of priorities.

Chen et al. [28] reported that the pairwise comparison method of the weighted layers will create a ratio matrix to view all the paired criteria and compare the different outputs, make changes to the input ranks and calculate consistency based on user's preferences . Sensitivity is determined by comparing dependency of the input parameters and the changed parameters to see if there is any significant impact on the final outputs.

\section{Conclusion}

Relatively little attention has been paid to how to conduct assessment of vulnerability of commercial property values to flooding in valuation or flood management research. In the light of changing flood risk and flood management policy in the UK it was found to be important to conduct such assessments in a way which not only reflects the current conditions but also helps to build capacity for the local communities by reflecting their views and opinions within the system. 
The structured approach of the proposed variation to the PVA methodology provides a well-focussed agenda, in the form of complete breakdown of the required tasks of vulnerability assessment and incorporation of information for both qualitative and quantitative options to improve transparency in the process.

The proposed method of spatial analysis integrates existing approaches and provides a spatio-temporal dimension by incorporating GIS. The methodology is in its developmental stage and anticipated to perform well if the data and other relevant information can be made available for analysis. The collection of data in proper GIS format might add complexity to the given approach; however with increasing level of data in digitised format, the development of appropriate approaches exploiting the potential of such data is encouraged. It is expected that an integrated purpose-built pluralistic assessment of flood vulnerability of commercial properties towards property values can be successfully performed involving stakeholders views in evaluation of alternatives for decision making in this field in future.

\section{References}

[1] House of Commons library research. Reducing Flood Risk. London. 2011.

[2] Kenny, S., Pottinger, G., F. and Yasmin, P., Flood risk and property,impacts on commercial \& residential stakeholders' strategy. College of Estate Management: Reading. 2006.

[3] Pagourtzi, E., Assimakopoulos, V., Hatzichristos, T., French, N., Real estate Appraisal: A review of valuation Methods. Journal of Property Investment and Finance, 21(4): p. 383-401. 2003.

[4] Shapiro, E., Davies, K., Mackmin, D., Modern Methods of Valuation. 10 ed. Malta: Melita Press. 701. 2009.

[5] Wyatt, P., Using a geographical information system for property valuation. Journal of Property Valuation and Investment, 14(1): p. 67-79. 1996.

[6] Thywissen, K., Components of Risk, in Studies of the University: Research, Counsel, Education- Publication series of UNU-EHS. UNU Institute for Environment and Human Security: Bohn. p. 52. 2006.

[7] ROOTS. The property implications of flood risk management, RICS practice information and guidance. in RICS rural research conference. Brighton. 2010.

[8] Environment Agency. Flooding in England: A national assessment of flood risk. Bristol. 2009

[9] UK Government. Flood and Water Management Act. London. 2010.

[10] Kenyon, W., Evaluating flood risk management options in Scotland: A participant-led multi-criteria approach. Ecological Economics: p. 70-81. 2007.

[11] Sybille, v.d.H., Participatory approaches to environmental policy-making: the European Commission Climate Policy Process as a case study. Ecological Economics, 33(3): p. 457-472. 2000.

[12] Environment Agency. Participatory risk assessment: Involving lay audiences in environmental decisions on risk. Reading. 2003. 
[13] Bhattacharya, N., Lamond, J., Proverbs, D., Flood vulnerability and hazard adjustment for UK commercial sector, in International Conference on Disaster Resilience: Srilanka. 2011.

[14] Vatsa, K.S., Risk, Vulnerability and asset based approach to disaster risk management International Journal of Sociology and Social policy, 24(10/11): p. 1-48. 2004.

[15] Zhang, Y., M.K. Lindell, and C.S. Prater, Vulnerability of community businesses to environmental disasters. Disasters, 33(1): p. 38-57. 2009.

[16] Balica, S. and N.G. Wright, A network of knowledge on applying an indicator-based methodology for minimizing flood vulnerability. Hydrological Processes, 23(20): p. 2983-2986. 2009.

[17] Balica, S., Wright, G. N., Reducing the complexity of flood vulnerability index. Environmental Hazards, 9: p. 321-339. 2010.

[18] Actionaid, Participatory Vulnerability Analysis - A step by step guide for field staff. Actionaid International: London. 2005.

[19] Penning-Rowsell, E.C., Wilson, T, Gauging the impact of natural hazards: the pattern and cost of emergency response during flood events. Transactions of the Institute of British Geographers, 31(2): p. 99-115. 2006.

[20] Messner, F., Meyer, V., Flood damage, vulnerability and risk perceptionchallenges for flood damage research, in Discussion paper. UFZ: Leipiz. 2005.

[21] Sigrid, S., Multicriteria evaluation and public participation: the case of UK energy policy. Land Use Policy, 23(1): p. 53-62. 2006.

[22] Prabhu, R., Sukadri, D., Purnomo, H., Hartanto, H., Guidelines for applying multi-criteria analysis to the assessment of criteria and indicators. CFOR: Jakarta. 1999.

[23] Petts, J., Homan, J., Pollard, S., Participatory Risk Assessment: Involving Lay Audiences in Environmental Decisions on Risk. Environment Agency: Reading. 2003.

[24] Muller, A., Reiter, J., Weiland, U., Assessment of urban vulnerability towards floods using an indicator-based approach - a case study for Santiago de Chile. Natural Hazards Earth System Science, 11: p. 21072011. 2011.

[25] Lamond, J.E., The impact of flooding on the value of residential property in the UK, in Dept. of build environment and engineering. University of Wolverhampton: Wolverhampton. p. 377. 2008.

[26] Edgar, M., Expert systems in investment decision-making. Estates Gazette. p. 62-64. 1994.

[27] Wyatt, P., The development of a property information system for valuation using a geographical information system (GIS). Journal of Property Research, 13(4): p. 317-336. 1996.

[28] Chen, Y., Yu, J., Shahbaz, K., Xevi, E. A GIS-Based Sensitivity Analysis of Multi-Criteria Weights in 18th World IMACS / MODSIM Congress. Cairns, Australia. 2009. 